\title{
De los métodos a la metodología en los diseños de investigación en ciencias sociales
}

\author{
From methods to methodology on social sciences research design
}

\author{
Diego Chaverri Chaves \\ Escuela de Sociología, Universidad Nacional, Costa Rica, \\ educarsociologia@gmail.com \\ Fecha de recepción: 5/08/2016. Fechas de reenvíos: 24/04/2017-17/05/2017, \\ 24/05/2017-25/05/2017. Fecha de aprobación: 12/09/2017. \\ Fecha de publicación: 28/09/2017.
}

\begin{abstract}
Resumen: En ciencias sociales se comparten los métodos y técnicas de investigación, esto supone acercamientos prácticos entre disciplinas. Este artículo ofrece un prontuario al respecto, de modo tal que señala la diversidad de métodos y técnicas, las cuales se potencian por su combinación e innovación, pero simultáneamente se advierten algunos errores frecuentes que merodean los diseños de investigación al construir las metodologías. La principal sugerencia es que cada nuevo proyecto construya una metodología para el caso específico, nunca basta con repetir procedimientos mecánicamente.
\end{abstract}

Palabras clave: método; metodología; técnicas; ciencias sociales; diseño de investigación.

\begin{abstract}
Social Sciences share research methods and techniques, which implies practical approaches between disciplines. A summary is offered in this paper including the diversity of methods and techniques, which are strengthened by their combination and innovation. The reader is also warned about some frequent mistakes made when constructing methodologies in research designs. The main suggestion is to construct a methodology for each specific case and every new project; repeating procedures mechanically should never be enough.
\end{abstract}

Keywords: method, methodology, techniques, social sciences, research design.

\section{Proceso de decisiones científicas}

Este texto puede ser propedéutico para el acompañamiento en el diseño de propuestas de investigación también conocidas como anteproyectos, no atiende el proceso completo de una investigación, sino sus etapas iniciales, y además ofrece un debate sobre la no siempre diáfana distinción entre métodos, técnicas y metodología en ciencias sociales, que son elementos fundamentales en esta etapa inicial. 
El punto de partida radica en reconocer la especificidad porosa y dinámica de cada disciplina del ramo, pero ninguna exenta de sujeción a prueba y consistencia lógica. Esto supone entonces que las técnicas de investigaciones y las opciones de método son compartidas por todas las ciencias sociales, la psicología no es la única que aplica encuestas, así como la antropología no es la única que ejecuta etnografías, ni los mapeos son exclusivos de la geografía, ni el análisis documental único en historia; desde tal arista las ciencias sociales concretamente comparten un acervo técnico instrumental de gran importancia y diversidad, es así también por vía de comparación como se logra precisar la especificidad de cada perspectiva en ciencias sociales, la cual yace en su teoría; aquí sí se puede señalar que las disciplinas hasta ahora mencionadas, y otras más del ramo, tienen sus teorías específicas, esto es lo que les permite definir sus objetos de estudio de manera diferenciada, tanto en su previsión como en su explicación. Luego se pueden trazar intercambios y también disputas entre distintas especialidades, en tanto no se puede presumir que las relaciones entre las ciencias derivan por fuerza en armonía, o que todas las teorías pueden concordar; más aún, la investigación científica logra utilidad en la localización de errores, para entender cómo estos se producen (Bourdieu et al, 2007).

Quizá una de las principales deficiencias del tipo de textos puramente metódicos es su desconocimiento de la fundamental importancia de la teoría, aunque coexisten con otros textos que sí recuperan esta faceta (Sautu, 2005); sin embargo, es tendencia todavía fuerte que algunos manuales de métodos y técnicas de investigación son ocasionalmente apresurados en el tratamiento de la articulación con la teoría, mientras otros textos, únicamente apuntan a la operacionalización de conceptos. Aquí se asume divergencia al respecto, la metodología logra su mayor coherencia conforme más avance teórico se logre desplegar, empero la tarea no se da fácil, mientras sí existe avance en una teoría del método, no ha sido tan elaborado el problema del método de la teoría, esto sugiere a quien investiga poner cuidado sobre cómo se incorpora su bagaje teórico en el proceso de diseño de una investigación en ciencias sociales, sin embargo esa discusión queda fuera de los alcances de este artículo. En síntesis, teorías y métodos se requieren entre sí, tomados aisladamente en los procesos de diseño de investigación pueden conducir a múltiples sesgos y equívocos.

En lo sucesivo, este documento se organiza a partir de los contenidos mínimos imprescindibles para una propuesta metodológica inicial de investigación lo suficientemente sólida y viable. Primero se discutirá la polémica sobre el método en ciencias sociales y luego su paso a la construcción de metodologías para el trabajo de campo así como para la sistematización de la información recabada, pasando revista a las técnicas de investigación.

Valga insistir una vez más, la teoría es vertebral en la definición del objeto de estudio, pero esto no siempre se ve reflejado en la redacción del diseño de investigación, y para lo que aquí interesa, una exposición teórica no puede reemplazar la indicación de la metodología, pues si no se corre el riesgo de convertir el anteproyecto en un ensayo teórico, desviando la atención de lo que se 
debe indagar y requiere de fuentes empíricas; quien diseña una propuesta metodológica para investigación ya debe tener bagaje teórico, pero aquella queda amputada si se reduce a señalar que se hará un abordaje fenomenológico, interaccionista, estructuralista, o constructivista, pues aún no precisaría metodológicamente.

Es debido reconocer que toda discusión metodológica es inservible si se presume autosuficiente; el avance no solo en el diseño de investigación, sino finalmente en su ejecución y finalización exitosa, depende de alguien con disposición para tomar decisiones inteligentes, informadas, críticas, para discernir dentro de las opciones de indagación; un mismo problema de investigación puede ser abordado con una u otra teoría, con algunas técnicas, desde cierta metodología, construyendo en cada caso diferentes objetos de investigación (y de variable calidad también), sin saber de antemano cuáles son las mejores opciones; pero si no se decide no se avanza, la clave está en lograr tomar decisiones con la mayor rigurosidad posible en el juicio.

El diseño de una investigación es siempre preliminar, está subordinado a una investigación todavía por hacerse ya sea para identificar patrones de conducta o relaciones, pronósticos, exploración de la diversidad social, o la inspección de casos (Ragin, 2007), de ahí que se torna difícil referirse a diseños de investigación acabados o finalizados, y esto hace a los mismos presa fácil de las sugerencias constantes por parte de muchos especialistas que son regularmente proclives a ofrecer alternativas para modificar el problema de investigación y lograr el planteamiento "verdadero" o "acertado", pero en realidad son indefectiblemente hipotéticos. Si no hay un trabajo exhaustivo sobre las fuentes disponibles el proceso será frágil, es decir, vulnerable a la indecisión y presa de recurrentes cambios en preguntas de investigación, temas, unidades de análisis y demás, lo cual obstruye cualquier avance sustantivo, estanca el proceso valorando innúmera cantidad de opciones potenciales.

Solo la investigación toda se termina, un diseño no ofrece conclusiones sobre un tema, por el contrario, se encarga de plantear interrogantes para indagación empírica, con sustento teórico y metodológico. La opción restante, la que aquí interesa, es esmerarse por lograr un diseño de metodología completo y congruente, es decir, cumple con requisitos de forma y contenido, plantea una pregunta de investigación racional y ofrece una alternativa viable para resolverla.

Lo anterior es finalmente un llamado a la modestia, ya que la investigación científica requiere dedicación, no es fácil producir nuevos hallazgos, pero tal desafío no es solamente una empresa individual, el avance todo de la ciencia es posible por la conjugación de esfuerzos colectivos por conocer, esa es la principal razón por la cual la metodología no es insufriblemente compleja. Además, contiene potencial heurístico (Gray et al, 2007), es decir, convoca a la creatividad de quienes investigan para resolver problemas de manera novedosa, pero esta destreza se logra con tiempo y dedicación a la investigación. 
Vol 37, N 55, (1-14), EISSN: 2215-2997, julio - diciembre, 2017

URL: www.revistas.una.ac.cr/abra DOI: http://dx.doi.org/10.15359/abra.37-55.4

\section{Rigor metódico para decidir la metodología}

La teoría sigue siendo orientadora aún, pues el problema planteado para el diseño metodológico se trabaja desde una teoría del método a la cual se subordinan los aspectos técnicos (Gutiérrez \& Brenes, 1977) que se verán más adelante, aquella consiste en desarrollar el problema de producir información válida para el conocimiento científico de lo social de manera minuciosa, refutable, fundamentada, siguiendo etapas de indagación documental, formulación de preguntas, recolección y análisis de datos (Ragin, 2007), no de manera mecánica, pero sí de forma tal que la experiencia de investigación sea susceptible de sistematización para ulteriores referencias, es decir, permite su continuación; esto es el método, un concepto más general, que continuamente aspira a tomar distancia de los mitos, sesgos, generalizaciones o familiaridad del sentido común, para lograr mayor objetividad repetibilidad, fiabilidad, precisión y validez en las ciencias (Gray et al, 2007).

Sin embargo, a pesar de tal generalidad, esta definición no está más allá de la polémica; enfáticamente, cuando se admiten varios métodos, las explicaciones de esta variabilidad no son unívocas. Una opción sería reducir el problema del método al tipo de inferencias, ya se trate de inducir o deducir, incluso se puede sumar la abducción (Rodríguez, 2005), o híbridos del tipo hipotético deductivo, pero esto presumiría que basta considerar el método como análisis del lenguaje científico (Gutiérrez \& Brenes, 1977), sin embargo las inferencias pueden ser parte tanto del diseño como del análisis.

Otro caso es el definir método por la técnica, de modo que la etnografía, la observación, o la entrevista serían métodos, esto es un error y una simplificación, si bien todo método debe incorporar técnicas para las fases de recogida de datos, no basta con esto, debe explicar cómo se ejecutan aquellas en función del problema de investigación, y además la investigación puede proponer el uso de una o varias técnicas.

Otra opción, también bastante frecuente, es definir el método según la disciplina; esto es, se supone que se tiene un método económico, uno histórico, uno psicológico, uno sociológico, un método antropológico, y así sucesivamente para cada disciplina, pero ya antes se señaló; las diferencias entre ciencias requieren mucho más de una elaboración teórica de sus objetos de investigación; al respecto incluso existen propuestas novedosas de métodos diversos en ciencias sociales que combinan, algo arbitrariamente aún, algunos de los criterios anteriores (i.e. Beltrán, 1994).

Otra organización que suele hacerse es entre investigar discursos o comportamientos, su formulación coloquial es la del tipo: "no es lo mismo lo que la gente dice a lo que la gente hace", esto es en buena medida cierto, pero no justifica una división tajante; se trata más bien de desentrañar el continuo tenso entre lo que se dice o se hace, las motivaciones de una 
acción pueden ser verbalizadas y llenarse de significados precisos (Giddens, 1997) y lo que resulta todavía más importante, el discurso es solamente otra forma de acción, sin embargo la separación puede ser útil para entender las incongruencias entre el testimonio de una institución o individuo y su conducta, o dicho de otra forma, estudiar las diferencias entre la "conducta verbal" y otros tipos de "conducta operante" (Skinner, 1981). De nuevo, las palabras son parte de una conducta, es decir, la investigación puede mostrar cómo las personas son más o menos conscientes de su situación social, pero no obvia que las fuentes de información pueden ofrecer tergiversaciones, mentir, olvidar, sesgar, guardar silencio, ignorar, (Bourdieu et al, 2007) etcétera. Así las cosas, luego se puede discernir cuáles son los procesos que anteceden las conductas humanas, y cuáles son sus resultados.

También es recurrente el debate, bastante superado en su dicotomía extrema, entre el método cualitativo y el método cuantitativo, en principio la cuantificación orienta los esfuerzos para contar, medir, analizar datos preexistentes, como los derivados de bases de datos, o producidos (en trabajo de campo), mientras que el abordaje cualitativo estudia con especial interés las maneras del comportamiento y el lenguaje como fuente de información, se interpretan conductas, testimonios, conceptos y creencias singulares, posibilita pormenorizar en casos, por tanto, la selección de las personas informantes o los lugares a investigar debe respaldarse en evidencias capaces de mostrar cuán sobresaliente es el caso elegido para ameritar tal nivel de detalle en la recolección de datos, ya sea que los informantes son clave, o los hechos tienen algo de insólito, paradigmático o invariable.

Ante la posibilidad de combinar paradigmas, es también oportuno señalar el falso dilema entre lo cualitativo y lo cuantitativo, en el fondo se trata de una división lógica, no ontológica de lo real, pensada para facilitar su primer abordaje (Alvira, 1983), esto sucede porque en realidad la cantidad es solamente una cualidad más de los objetos, sin embargo tan básica, tan elemental, que se ha especializado al nivel hoy conocido, en síntesis, las cualidades pueden ser cuantificadas con variable grado de precisión, pero también toda cantidad es un tipo de cualidad más o menos susceptible de registro en guarismo.

Finalmente, otros autores se inclinan por distinguir métodos según el tipo de estudio, con otras tantas alternativas. Una es la investigación aplicada cuando ensaya con diversas alternativas de solución para un problema social (Vargas, 2009), y se puede combinar incluso con dinámicas de involucramiento de los agentes sociales implicados en la investigación, como es el caso de la investigación acción participativa (Fals Borda, 2009).

Más composiciones del método son posibles al incorporar los estudios exploratorios o diagnósticos cuando la reunión de datos parte de una hipótesis que señala el desconocimiento empírico de un caso, esto guarda similitud con el trabajo experimental, fundamentalmente concentrado en fabricar condiciones que estimulen la reacción social, es decir, procura elaborar 
un cierto comportamiento de alguna manera inédito y registrar lo acontecido intentando controlar los detonantes de tal conducta. Aún quedarían las opciones de encauzar el método según se asuma de manera longitudinal, cuando a un mismo tema se le da seguimiento a lo largo de un tiempo y espacio más o menos extensos, como se suele hacer en algunos estudios de caso, o sino también de manera transversal, cuando se reconoce que un fenómeno se suscita de manera simultánea en diferentes escenarios, por tanto se indaga en varios a la vez, es decir, de manera comparativa (Venegas, 2008).

Las combinaciones de métodos son muchísimas, se podría diseñar una investigación que incorpore elementos de inferencia inductiva, con un enfoque cualitativo, un estudio de caso, con dinámicas de investigación acción, empero lo más destacado aquí es que tantas opciones son útiles para ofrecer resistencia a la rigidez metódica, por al menos dos razones; primera, no aceptar un purismo metódico que se muestra intransigente ante las condiciones sociales del objeto de investigación, impone un método únicamente por el carácter consuetudinario de su forma, por eso el método científico social no es un recetario de pasos a seguir de planteamiento del problema, experimentación y análisis de resultados, el movimiento supone varias direcciones y la necesidad de que todo diseño de investigación logre simultáneamente delimitar su problema y articularlo con el conjunto del proceso en que se inscribe (Zemelman, 1992); la segunda razón, porque el diseño también puede hacer propuestas metodológicas originales, estimulando así el carácter innovador de la ciencia ante una realidad siempre más compleja que cualquier método.

Todos los métodos científicos tienen invariablemente un componente descriptivo en tanto indagan aspectos empíricos puntuales sobre el devenir social, pero no pueden quedarse ahí, falta el salto científico para elaborar razones explicativas y comprensivas, por tanto, no se puede negar que un ingrediente que define en buena medida la calidad de una investigación es la persona investigadora en tanto logra perfilar teóricamente sus hallazgos, les da sentido en su exposición, lo social no se explica a sí mismo únicamente por las fuentes de información, sin importar cuáles son estas; la calidad de quien diseña una investigación se refleja en la construcción fundamentada de las decisiones para avanzar en el proceso de pesquisa.

En breve, el método, así en general, es un problema teórico y epistémico sobre las opciones arriba señaladas con énfasis en enfoques, tipos de investigación, inferencias, etcétera, siempre resultará abstracto en buena medida, pues su parte concreta es la metodología que consiste en la problematización aplicada a objetos de investigación precisos, elabora cómo se prevé el trabajo de campo, sus etapas, su incorporación de una o varias técnicas, la ejecución de cada una adaptada a las condiciones específicas del objeto de investigación y las características de las fuentes de información, es la metodología la que supera un llano discurso sobre la importancia del método, pues respalda las combinaciones de métodos y técnicas que se han elegido para el tema en cuestión, pero dicha mezcla es construida, eso quiere decir que requiere 
fundamentación. La metodología en un anteproyecto explica y problematiza la forma en que se procura obtener información para responder las preguntas de investigación (Ragin, 2007).

\section{Metodología y técnicas de investigación}

La concreción que requiere el trabajo de campo de una investigación pasa por establecer cuáles técnicas se utilizarán y cómo se aplicarán, de nuevo, el diseño de los instrumentos de cada una requerirá también respaldo teórico, e incluso ético, toda vez que podría significar el uso de un consentimiento informado, mediante el cual se establece que las personas informantes 0 las instituciones participantes están anuentes a brindar información sin por ello exponerse a algún tipo de censura o exhibición que pueda vulnerar su integridad, así como compromete a la persona investigadora a trabajar cuidadosamente, manteniendo el respeto necesario para el desenvolvimiento óptimo de la investigación, manejando con seriedad los hallazgos, y mostrando en el proceso su anuencia a concertar sobre las condiciones mismas en que se llevará a cabo la investigación, por ejemplo, con cuanta intensidad y frecuencia requerirá de la apertura de las personas colaboradoras para ejecutar el trabajo de campo.

Pasando a las técnicas las opciones son incluso mayores que las del método. Estas refieren a la manera en la cual la información del trabajo de campo será obtenida, para lo cual habrá que considerar primero cómo cada técnica elegida registra los hallazgos, de manera gráfica como con croquis o mapas, también con formas visuales y tangibles como documentos escritos, ya sea bitácoras de campo, o formularios de encuestas, grabaciones de audio en disco, medio digital u otro formato, e incluso la documentación fotográfica o con vídeo. Esto es importante, ya que los datos documentados posibilitan el acceso a la información a terceras personas, no directamente involucradas en el curso de la investigación, pero que ejercen revisión de pares de la misma, así como evita a quienes investigan tener que fiarse de su recuerdo individual que en ocasiones puede propiciar el sesgo de confirmación, el cual consiste en resaltar los datos que corroboran las hipótesis del trabajo, a la vez olvidando otra información que podría ofrecer contraste; para esto se debe analizar toda la información del trabajo de campo, no sólo fragmentos llamativos.

Algunas otras técnicas tendrán que diseñarse según su aplicación, pues varían las instrucciones de uso de cada una según se ejecuten a informantes en modalidad individual o grupal, pero además pueden ser instrumentos administrados por un tercero, por ejemplo encuestadores o camarógrafos, o tratarse de instrumentos auto-administrados, una encuesta resuelta directamente por el informante, o una tarea posterior a un taller grupal.

También las técnicas son más o menos participativas, esto según el nivel de involucramiento que tienen las personas informantes para plantear preguntas sobre el mismo tema, no solo para responder las preguntas ya preestablecidas por quien investiga, esto no debe confundirse con 
actividades participativas como se entiende en la modalidad de talleres sociales pensados para la resolución de problemas o en la forma de investigación-acción (en donde la participación es especialmente decisiva para las fases de acción), pues ingenuamente se podría considerar que las personas con opinar ya participan con su testimonio en el proceso de discusión sobre un conflicto que les interpela, pero eso no basta, pues si investigar significa interrogar, una investigación participativa supone que las personas informantes son capaces de plantear preguntas relevantes sobre lo social en cuestión y estas serán involucradas en el esfuerzo de estudio de quien investiga. No es fácil lograrlo, no siempre la población de informantes tendrá la capacidad de suministrar tal nivel de reflexión, supone en muchos casos que con anterioridad se han cuestionado a sí mismos, este es rara vez el caso, una investigación participativa no puede fundarse en visiones idílicas de la población informante o en ingenuidades morales que presumen que una investigación participativa es siempre mejor, recuérdese que la autonomía de las personas no es necesariamente violentada solamente por interrogarles por sus creencias y comportamientos, así como involucrarlas en la investigación no garantiza ampliar sus márgenes de libertad. Eso dicho, el mayor nivel de participación queda entonces como opción, no un imperativo, e igualmente requiere probidad metodológica si se incorpora.

Las técnicas también se pueden combinar, es importante definir si se utiliza una o varias, pero antes de suponer que la mezcla es siempre la mejor alternativa (sobre todo por replicar de manera miope el debate entre lo cualitativo y lo cuantitativo), es valioso considerar que cada técnica se debe aplicar con profundidad, nunca se justifica una técnica por presumir que las demás son superficiales, o que la elegida es verdaderamente profunda. La superficialidad o profundidad son cualidades de la investigación toda que finalmente evocan la capacidad de quienes realizaron la investigación, no es la técnica por sí sola ni por la combinación de muchas de estas; por el contrario, un error común es ofrecer el uso de tres o muchos más procedimientos, pero haciendo un uso demasiado apresurado de estos, imposibilitando explotar la capacidad de recabar datos que cada uno puede ofrecer. Es preferible entonces la exhaustividad con cada técnica elegida, antes que una multiplicidad superficial de ellas. Evidentemente lo anterior también supone considerar los recursos disponibles, cada técnica toma su tiempo y esmero, involucra ciertos gastos, no se debe decidir a la ligera sobre esto, la exhaustividad en una técnica en ocasiones se puede lograr por saturación, cuando la información recopilada se empieza a repetir de manera más o menos regular, o también se puede lograr por censo o muestreo, cuando se agota la diversidad de fuentes de información disponibles o se abarcan en la mayor medida posible.

La mezcla de técnicas es otra alternativa, igual requiere ser construida, no es vía eclecticismo, de modo que la clave radica en considerar qué aporta cada una, cómo esto puede facilitar o dificultar el tratamiento de las fuentes de información, el nivel de exhaustividad que puede lograr, pero sobre todo la pertinencia con el objeto de investigación y las preguntas que se le hacen. La mixtura puede ser una vía de innovación en los procedimientos y métodos de 
investigación, pero no es garantía de mejores resultados en el trabajo de campo, por eso es importante considerar en un diseño de investigación que si la propuesta combina técnicas diferentes, debe ser la metodología la que considere los alcances y limitaciones para el trabajo de campo, cada nueva técnica puede ampliar la indagación de la realidad social, pero también impone sus condiciones, y amplía la cantidad de problemas prácticos que resolver.

Adicionalmente, para buscar congruencia en la muy frecuente combinación de técnicas en la investigación en ciencias sociales, aquellas pueden orientarse congruentemente a través de un cuestionario matriz, este consiste en una batería de preguntas genéricas que traducen el problema de investigación en interrogantes empíricamente accesibles, orientadas y adaptadas a las características de las fuentes de información. Esta matriz brinda la oportunidad de generar preguntas puntuales para cualquier técnica, logra clarificar aquello que se quiere obtener en el trabajo de campo, aunque esta matriz rara vez forma parte del diseño, es útil para quien investiga, pues puede colaborar en la toma de decisión sobre la técnica de investigación idónea, esto quiere decir que un grupo focal, una observación, o un mapeo, están orientados por preguntas de investigación derivadas de los objetivos centrales, así se puede obtener un panorama de los aportes de cada técnica, y mostrar también que pueden dejar desatendido.

Cada técnica a su vez tiene su propia complejidad, pero este trabajo no las agota, de tal manera los tipos de preguntas requieren profundizar en la revisión de la particularidad de cada técnica, por ejemplo el uso de reactivos, preguntas disyuntivas, escalas, o respuesta múltiple, así con un amplio margen de opciones; una vez que en el diseño se decida sobre las técnicas óptimas para el trabajo de campo, es prudente la revisión bibliográfica para ponerse al tanto sobre las minucias de las mismas, en este documento únicamente se ofrece un repertorio de opciones y algunos criterios para decidir de manera práctica sobre su uso, pero no se profundiza en cada procedimiento con el detalle que merece.

De una misma técnica se pueden derivar uno o varios instrumentos; por ejemplo, si se realiza una entrevista, podrán existir diferentes versiones según el perfil de los informantes entrevistados, también un grupo focal puede tener un instrumento de trabajo distinto para cada subtema u objetivo de la investigación, esto es importante tenerlo claro, una técnica no se agota en un solo instrumento.

Sobre cada instrumento es prudente ver la variación según el tipo de preguntas, y también la secuencia que pueden tomar las mismas, lo que se expone a continuación no supone un orden mecánico, sino distintos intereses de los instrumentos que permiten reflexionar sobre cuáles deben ser los primeros temas que se indaguen, cuáles deben ser los últimos, o también cómo se pueden intercalar. Esto requiere algún primer conocimiento de las cualidades de las fuentes de información, intentar prever sobre cuáles aspectos puede ser más fluida la recolección de datos y dónde se pueden presentar discreciones o hasta prohibiciones (recuérdese que la respuesta a las preguntas puede ser por observación de conducta, registro de fuentes documentales, o por interrogación verbal al informante). 
Las preguntas introductorias se encargan de obtener las principales características de la persona o institución que brinda información en el trabajo de campo, esto puede apuntar a rasgos como su origen, el alcance espacial o temporal de sus aportes, y especialmente cuando la fuente de información es una persona, identifica su perfil demográfico relevante, como el sexo, edad, nivel de escolaridad, estrato ocupacional, entre muchas otras según lo que interese al estudio.

Las preguntas testimoniales son del tipo que permiten observar el conjunto de información que la fuente puede brindar desde su propia óptica o experiencia, se trata de la experiencia de lo que alude al tema interrogado en la investigación del caso, es decir, los informantes podrán ser estudiados desde lo que hacen o dicen, esto incluye a los grupos o instituciones, puede tratarse por ejemplo de comunicados oficiales de una organización, recopilados por quien investiga.

Luego las preguntas de problematización, solo se incluyen en los instrumentos cuando se espera que la fuente de información pueda profundizar sobre los datos previamente brindados a nivel testimonial, e indique limitaciones o problemas, se confronta a la fuente de información con dilemas o conflictos derivados de su conducta, ya sea obtenida con bastante anterioridad o en etapas previas de la aplicación del instrumento; a nivel de comportamiento también se logra con trabajos longitudinales al verificar como el objeto se comporta en distintos escenarios, ya sea que varíen las circunstancias o los agentes involucrados.

Las preguntas de control consisten fundamentalmente en la reformulación de las anteriores para verificar la confiabilidad de la información obtenida. Esto se aplica cuando existen razones para sospechar que la fuente puede tergiversar información, lo cual se logra al establecer cruces entre los datos obtenidos, de tal manera que también se puede definir la influencia de factores que podrían no estar siendo estudiados; algo parecidas pueden resultar en ocasiones las preguntas de evaluación, para identificar el nivel de conocimiento o destreza.

Una opción más son las preguntas residuales; con éstas se busca considerar el aporte general de la fuente de información, e incluso admite espacios para anotar datos no esperados o nuevas preguntas, así como confusiones que habrían surgido durante la aplicación del instrumento. El propósito es que después de aplicar algunos tipos de instrumento, muy probablemente la fuente ha sido cuestionada sobre aspectos hasta ahora desapercibidos, lo cual eventualmente puede o no desencadenar procesos de reflexión inéditos, y originen nuevas conductas, pensamientos o interrogantes.

De lleno en las técnicas disponibles, a continuación se ofrece un breviario de estas, tampoco exentas de polémica, pues algunas se presumen métodos. Aquí el señalamiento es provisionalmente simple, las técnicas se definen según cómo producen la recuperación de información en campo, aquí entonces se indica cuál puede ser al menos uno de los intereses para decantarse por el uso de una técnica sobre otra, nótese una vez más, nunca se presume que 
la técnica es profunda o superficial y meramente descriptiva, estas son cualidades supeditadas a quien investiga; hay formas superficiales de aplicar cualquier técnica, así como se pueden orientar con mayor aplomo y congruencia; de nuevo, la profundidad puede sugerirse desde la teoría del diseño y las cualidades de quienes investigan. Las técnicas siguientes no son todas las disponibles, pero sí suelen ser de las más recurridas en la investigación en ciencias sociales, se pueden combinar y cada una varía según las cualidades que se discutieron arriba, como la aplicación, el tipo de preguntas, la secuencia y la metodología en que se inscriben, esto supone que una técnica por sí sola no pertenece de manera exclusiva a un método, la encuesta se puede aplicar para uno comparativo, así como la entrevista puede ser utilizada dentro de uno longitudinal, tampoco se distingue ingenuamente entre técnicas cuantitativas y cualitativas por las razones ya mencionadas arriba, cada técnica debe ser diseñada para guardar congruencia con la teoría y la metodología, no es un proceso mecánico, requiere decisiones científicas. Obsérvese a continuación una sinopsis de algunas de las técnicas de investigación más frecuentes en ciencias sociales:

- Entrevista: recupera el testimonio de informantes clave, puntualiza sobre sus conocimientos del tema, les permite explayarse. Hay una diversidad importante de tipos de entrevista, según los temas, su dinámica de aplicación, o la definición de los informantes clave.

- Historia de vida: reconstruye la biografía de una persona a través de varias fuentes, por ejemplo datos genealógicos, entrevistas a familiares o colegas, entre otras. Esta técnica suele ser muy cercana a la entrevista biográfica, pero no depende de esta, pues la historia de vida se podría hacer incluso sobre un grupo social o una persona ya fallecida.

- Encuesta: sistematiza información equivalente en una misma población, de forma tal que los instrumentos deben habilitar cierto nivel de estandarización en las preguntas en cada ítem y las respuestas obtenidas.

- Observación: caracteriza escenarios de interacción, define fronteras de tiempo y espacio de observación, documenta prácticas, quien investiga puede o no involucrarse en éstas últimas. En ocasiones se puede asemejar al mapeo, que es la realización gráfica de espacios geográficos o institucionales, puede recurrir a organigramas, flujogramas, croquis o incluso implementar sistemas de información geográfica.

- Análisis de discurso: indica orientaciones que toman discursos singulares, aunque estos no suelen ser propiciados por quien investiga, solo recopilados en ocasiones in vivo. Por su parte el análisis de contenido: muestra tendencias de un grupo de fuentes documentales, muy similar a la técnica anterior, suele variar por rastrear un tema en una diversidad de fuentes, ya sean textuales, fotográficas, auditivas, o de video, en formato físico o digital. 
- Sociometría: mide la intensidad de vínculos entre personas u organizaciones, se puede valer muy comúnmente de la aplicación de algún tipo de test. En ocasiones se puede expresar gráficamente, abriendo campo al sociograma, como una técnica que indica tipos de vínculos entre personas $u$ organizaciones y sus dinámicas, también puede ser referido como el análisis de redes y graficarse en consecuencia.

- Sociodrama: manifiesta emociones y relaciones de grupo sobre temas dados, permite la reconstrucción de testimonios o actitudes en escenarios hipotéticos mediante la dramatización.

- Representación social: visibiliza imaginarios o prejuicios de una población, documenta las reacciones a diversos temas, a través de objetos, imágenes o palabras.

- Grupo focal: reconstruye escenarios de discusión de grupos, a diferencia de la entrevista meramente grupal permite capturar la conversación de informantes entre sí y sus reacciones

- Experimento: procuran registrar la variación de factores que pueden detonar o inhibir un comportamiento, al igual que en el estudio de representaciones es común el uso de reactivos, puede tener estrategias de experimentación hipotética, o de reconstrucción de un escenario, tipo laboratorio.

- Etnología: suele ser una combinación de varias de las técnicas anteriores, pero aplicadas siempre in situ, lo cual en su sentido clásico supone la observación participante, pero con énfasis en el estudio comparado de culturas, mientras que la inmersión en un campo cultural específico es más propio de la etnografía, así como el estudio de grupos culturales originarios es propio de la etnohistoria que suele recurrir a técnicas ya antes mencionadas y otras como la genealogía, para recuperar troncos de progenies.

\section{La metodología y los problemas de investigación}

Una metodología solamente se obtiene cuando se han fundamentado, en función del objeto de investigación, las decisiones sobre las opciones de métodos y técnicas para su aplicación concreta en el trabajo de campo y su posterior sistematización, la metodología debe delimitar, al decidir sobre posibilidades de métodos y técnicas, también al enfocar en tiempo y espacio el tema, explica sus etapas de incursión, establece poblaciones de informantes o contextos, proyecta alcances y por tanto define el nivel de exhaustividad esperado. La metodología explora los principios, procesos y estrategias de investigación (Gray et al, 2007), es la construcción de un método y su fundamentación para una investigación concreta, no es discurso abstracto, es teoría del método aplicada a una propuesta específica. 
Por su parte, el problema de investigación no emerge espontáneamente sino con trabajo metódico, requiere estudio de fuentes y del tema en cuestión; la elaboración sistemática de una pregunta de investigación supone resolver quién pregunta, cómo se pregunta, cuándo se pregunta, dónde se pregunta, de modo que la persona investigadora pueda ofrecer una interrogante contextualizada que sea viable responder en el marco de un proyecto (Andrews, 2003); no se pregunta todo, ni se pregunta cualquier cosa.

Es sensato advertir que una buena investigación no se reduce a la única alternativa del descubrimiento científico ni a sus derivaciones "técnicas", en ocasiones el modesto trabajo de descartar otras vías de exploración, o comprobar fallos, ya es un aporte significativo, y en ocasiones hasta más preciso, pues reconoce sistemáticamente los errores, añade al acervo del conocimiento de lo social como una composición espesa y múltiple. Además, la metodología es también útil para discernir por qué en ciencias sociales algunas investigaciones discrepan o concuerdan, así como para valorar cuáles resultados lograron mayor precisión y acierto (Gray et al, 2007).

En ciencias sociales, ante la multiplicidad de métodos y técnicas será cada vez más arduo contar con un vademécum, pero a la vez esto significa una gran apertura a recomendaciones de diverso tipo; las metodologías se construyen para cada tema por trabajar, no se implantan, sino que consideran todas las variables reseñadas en este breve artículo. Además de metodologías precisas, la organización y sistematización del trabajo de campo permiten que aquellas resulten auto-críticas, habilitando la revisión de errores y la asignación de limitaciones en todo el proceso de transformación de los datos en conocimiento científico social. El diseño está en las etapas iniciales de un ciclo de investigación, requiere momentos para revisar y corregir continuamente las preguntas, técnicas, y fuentes de información, de ahí que una metodología rigurosa en el arranque favorece la identificación de aciertos y desaciertos en el proceso tanto como en su cierre, favorece la capacidad de auto-crítica de quienes investigan, para así tener mayor destreza para el diseño de la siguiente pregunta de investigación y su metodología.

\section{Referencias bibliográficas}

Alvira, F. (1983). Perspectiva Cualitativa - Perspectiva Cuantitativa en Sociología. En: Revista Española de Investigaciones Sociológicas. Número 22. Centro de Investigaciones Sociológicas. España.

Andrews, R. (2003). Research Questions. Continuum, Estados Unidos.

Beltrán, M. (1994). Cinco vías de acceso a la realidad social. En: García, Manuel; et al. (compiladores). El análisis de la realidad social. Métodos y técnicas de investigación. Alianza Universidad Textos. España. 
Bourdieu, P.; Chamboredon, J.C.; Passeron, J.C. (2007). El oficio de sociólogo. Siglo XXI Editores. España.

Fals Borda, O. (2009). Una sociología sentipensante para América Latina. CLACSO, Siglo del Hombre Editores, Colombia.

Gallardo, H. (2008). Fundamentos de Investigación Académica. Editorial Universidad Estatal a Distancia. Costa Rica.

Giddens, A. (1997). Las nuevas reglas del método sociológico: crítica positiva de las sociologías comprensivas. Amorrortu Editores. Argentina.

Gray, P.; et al (2007). The Research Imagination. An introduction to qualitative and quantitative methods. Cambridge University Press, Inglaterra.

Gutiérrez, C.; Brenes, A. (1977). Teoría del método en las ciencias sociales. Editorial Universitaria Centroamericana. Costa Rica.

Ragin, C. (2007). La Construcción de la Investigación Social. Siglo del Hombre Editores -Universidad de los Andes, Colombia.

Rodríguez, R. (2005). Abducción en el contexto del descubrimiento científico. En: Revista de Filosofía. Número 109/110, Universidad de Costa Rica, Costa Rica.

Sautu, R. (2005). Todo es teoría: objetivos y métodos de investigación. Ediciones Lumiere, Argentina.

Skinner, B. (1981). Conducta verbal. Editorial Trillas. México.

Vargas, Z. (2009). La investigación aplicada: una forma de conocer las realidades con evidencia. En: Revista Educación, vol. 33, n. 1. Universidad de Costa Rica, Costa Rica.

Venegas, P. (2010). Algunos elementos de investigación. Editorial Universidad Estatal a Distancia. Costa Rica.

Zemelman, H. (1992). Los horizontes de la razón: Uso crítico de la teoría. Editorial Anthropos, México. 